\title{
Implications of inconsistent anaemia policies for children and adolescents in Africa
}

\author{
Musa Hamdan ${ }^{1}$, Bernard Brabin ${ }^{2}$ and Imelda Bates ${ }^{3, *}$ \\ ${ }^{1}$ Medicins Sans Frontières, Amsterdam, The Netherlands: ${ }^{2}$ Tropical Paediatrics, Liverpool School of Tropical \\ Medicine, Liverpool, UK: ${ }^{3}$ Tropical Clinical Haematology, Liverpool School of Tropical Medicine, Pembroke \\ Place, Liverpool L3 5QA, UK
}

Submitted 29 March 2013: Final revision received 15 0ctober 2013: Accepted 20 0ctober 2013: First published online 28 November 2013

\begin{abstract}
Objective: To evaluate the quality of policies concerning the diagnosis, treatment and prevention of anaemia in children and adolescents; to determine to what extent these are evidence-based; and to use this analysis to inform the policymaking process.

Subjects: Children and adolescents in sub-Saharan Africa.

Setting: Almost 50\% of children and adolescents in sub-Saharan Africa are anaemic, which has profound effects on their intellectual and physical development and their chance of survival. Evidence-based policies are essential to reduce anaemia but because it is caused by an array of interdependent factors, developing policies is challenging.

Design: Forty-six policy documents concerning the diagnosis, treatment and prevention of anaemia in children and adolescents were identified and analysed. Results: There was policy consensus on the usefulness of Fe supplements, the need to treat co-morbidities and the use of blood transfusions for severe anaemia. Information about diagnosis was scarce, and messages regarding the control of anaemia were mixed. Few of the policies were tailored for the African context and they were located on several websites hosted by different health programmes.

Conclusions: The weakest aspects of the policies and consequently the priorities for better policy making were: lack of adherence to WHO recommendations for guideline development; little involvement of African practitioners/policy makers in the guideline group and as peer reviewers; and lack of harmonisation, demonstrating the need to establish a single body responsible for developing/ revising anaemia policies.
\end{abstract}

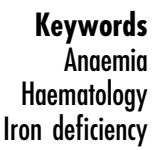

Keywords

Haematology

Iron deficiency
In sub-Saharan Africa, the high prevalence of infectious diseases such as malaria, helminthiasis and HIV, chronic diseases including haemoglobinopathies and deficiencies of micronutrients such as Fe, cobalamin, folate and vitamin A are responsible for causing anaemia in an estimated $47 \%$ of children and adolescents ${ }^{(1,2)}$. This panoply of interdependent factors is a major challenge for developing clear, cohesive and evidence-based policies for anaemia diagnosis, prevention and treatment. Adapting these policies to make them applicable for resource-poor settings in Africa presents an additional challenge $^{(2)}$. International anaemia policies relevant for children in Africa are produced by several agencies and by different units within single agencies; for example, those dealing with malaria, HIV, nutrition and neglected tropical diseases including helminthiasis (also see Box 1). The wide variety of diagnostic methods, treatment approaches and prevention programmes for anaemia in various risk groups that are practised in Africa suggests that the guidance offered by current policies may be inconsistent, or difficult to apply, in the African setting. An additional complication is the fact that the definition of anaemia in countries varies with age and sex; for example, from $\mathrm{Hb}<94 \mathrm{~g} / 1$ in infants aged 2 months to $\mathrm{Hb}<130 \mathrm{~g} / 1$ in male adolescents. In the present study we have identified and compared international policies concerning anaemia diagnosis, treatment and prevention in children. We have evaluated the quality of these policies and the extent to which they are based on evidence relevant to the African context, and have made recommendations for improvements to the policy-making process. We used the WHO definition for 'guidelines' to identify relevant policies and therefore included 'documents that contain recommendations about health interventions, whether they be clinical, public health or policy interventions'. 


\section{Box 1}

\section{Identification of relevant documents concerning anaemia diagnosis, treatment and prevention in African children}

Databases and websites interrogated for documents

- WHO databases: Archives of WHO; WHO Global Health Library; Bulletin of the World Health Organization; WHO Reproductive Health Library.

- WHO programmes: Blood Transfusion Safety; Nutrition; Child and Adolescent Health and Development; WHO Diagnostics and Laboratory Technology; HIV/AIDS; Malaria; Neglected Tropical Diseases; Tuberculosis.

- WHO special programmes/partnerships: Partnership for Maternal, Newborn and Child Health (MCH); Tropical Diseases, Special Programme for Research and Training (TDR); Stop TB Partnership; Roll Back Malaria.

- UNICEF website.

- Micronutrient Initiative website.

- National Institutes of Health (USA).

- Other references from bibliographies in above documents and through personal contacts.

Search terms and definitions used to identify and select documents

Search terms (alone or in combination): 'anaemia', 'anemia', 'diagnosis', 'screening', 'treatment', 'prevention', 'control', 'recommendation', 'strategy', 'children', 'Africa'.

\section{'International'}

- Any document/publication issued by internationally recognised health bodies such as WHO and UNICEF (including regional offices for Africa).

- Any document/publication by an international programme concerned with anaemia (e.g. Nutrition, MCH, HIV, Tuberculosis, Neglected Tropical Diseases, Malaria, Micronutrient Initiative and Blood Transfusion programmes).

'Policy'

Any guideline, recommendation, statement, technical information, report or expert opinion concerning the diagnosis, treatment and prevention of anaemia in children in Africa.

\section{Methods}

\section{Identification of international anaemia policies relevant for African children and adolescents}

Databases and websites of international policy-making organisations (Box 1) were searched for documents relevant for the diagnosis, treatment and prevention of anaemia in children and non-pregnant adolescents (i.e. $<18$ years old) in Africa (Fig. 1). No restrictions on language or time period were applied; the search was completed in May 2013. Relevant documents were identified from their abstracts and data were extracted using a pre-tested form. Although our strategy for identifying relevant policies was comprehensive and covered websites and documents likely to be accessed by policy makers and clinicians, it is possible that we may have overlooked some documents.

\section{Quality of international anaemia policies relevant for African children and adolescents}

The Appraisal of Guidelines for Research and Evaluation instrument (AGREE II) has been recommended by WHO for development of guidelines ${ }^{(3)}$ and was used without any adaptations to assess the quality of the policies ${ }^{(4)}$. This instrument uses twenty-three questions grouped into six domains (scope and purpose, stakeholder involvement, rigour of development, clarity of presentation, applicability and editorial independence) to score quality on a scale of 1-7. After converting the scores to percentages, the policies were categorised as very low quality (0-25\%), low (26-50\%), medium (50-75\%) or high quality (76-100\%) for each of the six domains (Table 1). The fifth domain of the AGREE II instrument addresses the general applicability of the policy rather than its specific application to children in Africa. The questions in the fifth domain focus on information about facilitators and barriers to their application, the provision of advice or tools for implementation, consideration of resources implications, and information about monitoring and auditing criteria.

\section{Identification of evidence concerning anaemia diagnosis, treatment and prevention in African children and adolescents}

The Cochrane Library database was searched using the terms in Box 2 for reviews and trials about anaemia 
Box 2

Agreements and discrepancies between policies and evidence concerning anaemia diagnosis, treatment and prevention

\section{Agreements}

- Fe supplements are generally beneficial for treating anaemia.

- Blood transfusion is indicated in children with severe anaemia who are clinically unstable.

- Clinical diagnosis of anaemia is unreliable for mild and moderate anaemia.

- Co-morbidities (e.g. worms) should be treated alongside the anaemia.

- Fe supplementation should be delayed in malnutrition.

- An integrated approach is needed for preventing anaemia.

- Fe supplementation should be delayed in the neonate.

- In malarious areas, good malaria surveillance and control should be provided for children receiving Fe supplements.

\section{Discrepancies}

- Definitions of anaemia and of grades of severity.

- Reliability of clinical diagnosis of anaemia.

- Variations in recommendations by age group.

- Relationship between Fe supplementation and increased risk of infection (and hospital admission), especially in the presence of malaria or HIV infection.

- Relationship between Fe supplementation, infection risk, and anaemia treatment and prevention in sickle cell disease.

diagnosis, treatment and prevention in children in Africa. Evidence to underpin anaemia policies may be available outside the Cochrane Library and other types of research, such as qualitative studies, may also be important for policy making. However, our study was not a comprehensive review of anaemia research. Instead we wanted to indicate broadly whether or not the policies were based on a significant body of evidence, so we focused on the high-quality evidence available in the Cochrane Library. A summary of evidence related to anaemia diagnosis, treatment and prevention in children is Africa is listed in the online supplementary material (Supplemental Table 1) and summarised in the third subsection of the Results.

\section{Results}

\section{Analysis of international anaemia policies relevant for African children and adolescents}

A total of 1247 documents were identified and screened and forty-six were selected for analysis. Reasons for excluding 1201 documents were that they were not relevant to the topic or Africa, not policy documents, not from a recognised international organisation, were duplicates or were unobtainable (Fig. 1). Forty-one of the forty-six documents were produced by WHO either alone or in collaboration with other agencies; and of these, only three were from WHO regional offices in Africa and the Eastern Mediterranean. Of the nine policies produced after 2008 when the first WHO Handbook for Guideline
Development was published, only one has been updated. Various units in WHO had produced the documents including units dealing with child and adolescent health, nutrition, blood transfusion safety and soil-transmitted helminths. The documents included guidelines and recommendations, expert opinions/consultations and statements $^{(2,5,6)}$. The target audience (e.g. health provider, policy maker, programme manager, nutritionist, public health official) was specified in thirty-five documents. Twenty-three of the documents did not specify the age group of children at which the policy was targeted. Policies covered urban and rural settings, and hospital-, home-based and community interventions.

Twenty-two policies did not define anaemia and where it was defined, the cut-off levels were inconsistent between documents. For example, the definition of severe anaemia varied from $\mathrm{Hb}$ level $<40 \mathrm{~g} / \mathrm{l}$ to $<75 \mathrm{~g} / \mathrm{l}$. Recommendations concerning treatment for anaemia appeared in twentyseven policies; Fe supplementation was mentioned in almost all of the policies, often in combination with other interventions such as blood transfusion, micronutrients, anthelmintics and antimalarials. Cautions about potential interactions - for example, between Fe supplements and infections, and between folate and antimalarials - were provided inconsistently between policies. There was reasonable agreement between policies on the need for an integrated approach to anaemia prevention consisting of Fe supplementation and food interventions combined with malaria control, hookworm control, and management of underlying conditions and co-morbidities. 
1247 documents titles and abstracts were screened

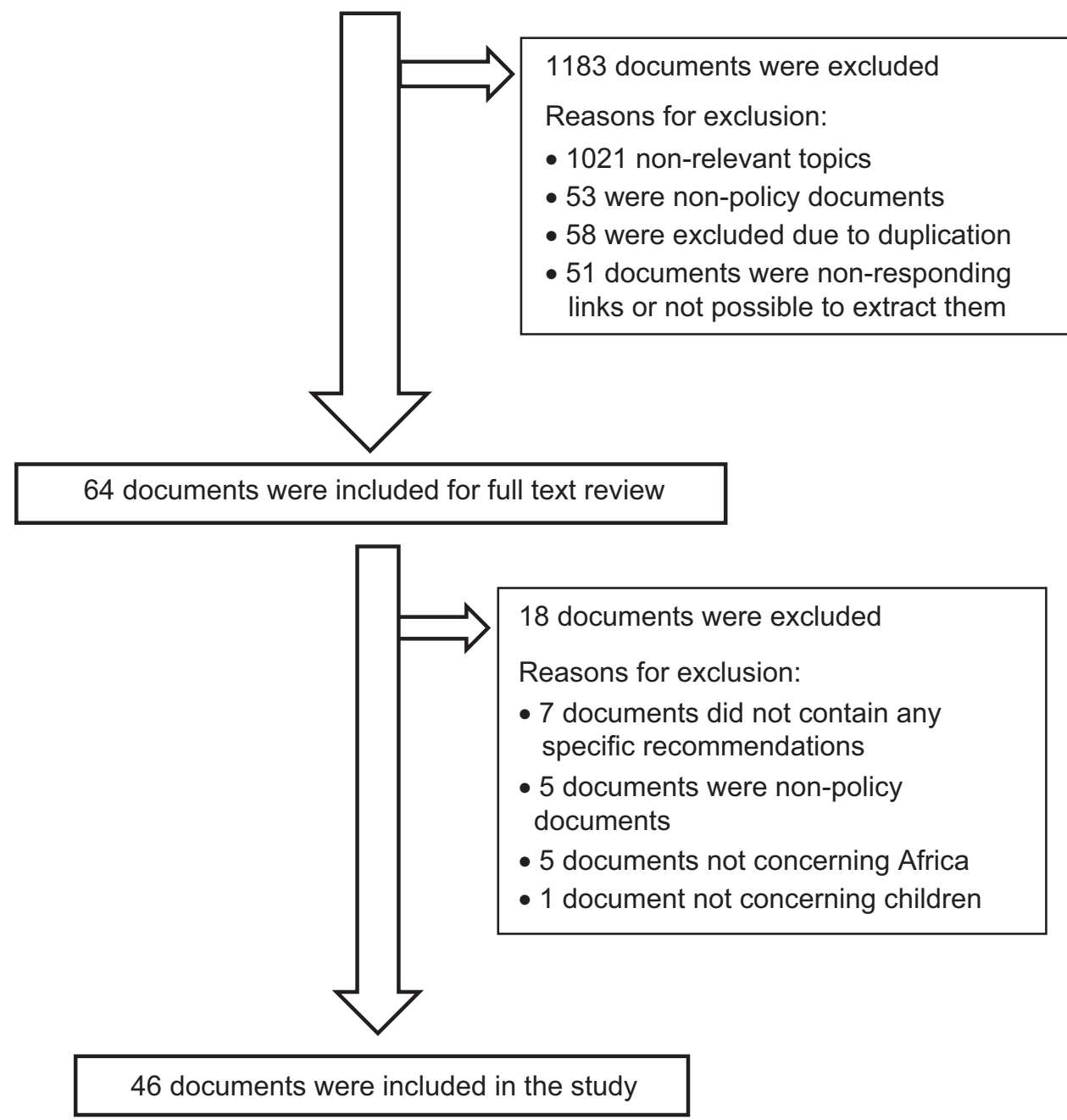

Fig. 1 Selection process for documents

Table 1 Number of policies (total of forty-six) in each quality category assessed against six quality domains

\begin{tabular}{lrrrc}
\hline & \multicolumn{4}{c}{ Quality of policy } \\
\cline { 2 - 5 } Quality domain & High & Medium & Low & Very low \\
\hline 1. Scope and purpose & 35 & 6 & 4 & 1 \\
2. Stakeholder involvement & 0 & 24 & 15 & 7 \\
3. Rigour of methods & 6 & 1 & 17 & 22 \\
4. Clarity of presentation & 29 & 10 & 4 & 3 \\
5. Applicability & 9 & 18 & 8 & 11 \\
& & & & \\
\cline { 2 - 5 } & & & & \\
6eported & & Not reported \\
\hline
\end{tabular}

\section{Quality of international anaemia policies relevant for African children and adolescents}

Twenty-seven of the policies had medium or high scores for the quality of their applicability and nineteen had low or very low scores. Four policies ${ }^{(7-10)}$ had average scores for the six domains that exceeded $75 \%$ and were considered to be of high quality. Twenty-seven of the policies (59\%) had average scores of less than $50 \%$ and were therefore of low or very low quality. Overall the weakest components of the policy development were the lack of stakeholder involvement and the rigorousness of the methods (domains 2 and 3), because recommendations were not explicitly underpinned by evidence (Table 1 ).

\section{Evidence available to inform policies concerning anaemia diagnosis, treatment and prevention in African children and adolescents}

Two hundred and thirty-two publications were identified which may have contained evidence to guide anaemia policies and twenty-seven included relevant information for our study and were eligible for analysis. Fourteen were systematic reviews and thirteen were randomised controlled trials. The paragraphs below provide an outline of the key findings. 


\section{Diagnosis}

Diagnosis was covered by only two reviews and was therefore the most neglected aspect of anaemia research. The reviews highlighted the poor accuracy of clinical signs in detecting anaemia in children ${ }^{(11)}$ and the need for more 'real-life' studies to evaluate the $\mathrm{Hb}$ colour scale for use where there was no laboratory ${ }^{(12)}$.

\section{Treatment}

Treatment was covered by seven publications which examined Fe supplementation, blood transfusion, anthelmintics and vitamin A supplementation in children. Fe deficiency is believed to contribute about $50 \%$ of anaemia but this may not be an accurate estimate as the terms 'anaemia', 'iron deficiency' and 'iron deficiency anaemia' were often not clearly defined. The evidence suggested that improvements in $\mathrm{Hb}$ as a result of $\mathrm{Fe}$ supplements are significant but modest and lower in regions with high malaria transmission ${ }^{(6)}$. Evidence suggested that there was no need to screen for anaemia prior to supplementation, although information on baseline $\mathrm{Hb}$ was limited ${ }^{(13)}$. Evidence did not support the use of Fe supplementation in HIV patients ${ }^{(14)}$ or in situations where there was poor malaria surveillance and control ${ }^{(2,14)}$, and it did not support the use of transfusions in clinically stable children with severe anaemia ${ }^{(15)}$. Albendazole in combination with praziquantel appeared effective for improving $\mathrm{Hb}$ levels ${ }^{(16)}$, and vitamin $\mathrm{A}$ supplementation stimulated erythropoiesis in children with severe anaemia ${ }^{(17)}$.

\section{Prevention}

Prevention was covered in ten reviews and eleven trials. Fe supplementation had no detrimental effect on child morbidity ${ }^{(18)}$ and it seemed more effective than food fortification $^{(19)}$ and more efficient when combined with malaria treatment and other health measures ${ }^{(20,21)}$. Home fortification of foods with multiple micronutrient powders is an effective intervention to reduce anaemia and $\mathrm{Fe}$ deficiency in children aged 6-23 months ${ }^{(22)}$. However, data on effects on malaria outcomes are lacking except for one Ghanaian trial which showed that in a malariaendemic setting in which insecticide-treated bed nets were provided and appropriate malaria treatment made available, daily use of micronutrient powder with Fe did not result in an increase in malaria incidence among young children ${ }^{(23)}$. A trial of multiple micronutrient powders among Pakistani children reported significant excesses of bloody diarrhoea and respiratory indrawing which may have been related to the Fe in the powder ${ }^{(24)}$. In some studies Fe supplementation improved $\mathrm{Hb}$ levels without worsening other infections including malaria ${ }^{(25)}$, but one large study of 32000 young children ${ }^{(26)}$ found that it increased the risk of severe disease and death in a malaria-endemic region. Another study ${ }^{(14)}$ found no benefit of Fe supplementation for HIV-infected children.
Overall, the risk for clinical malaria was higher with Fe, or with Fe plus folic acid, in trials where services did not provide for malaria surveillance and treatment ${ }^{(13)}$. Other trials have demonstrated increased adverse effects ${ }^{(27)}$, including increased hospital admissions ${ }^{(23)}$. Other useful measures assessed for reducing anaemia included malaria control ${ }^{(20,28,29)}$, anthelmintics ${ }^{(30)}$ especially albendazole-praziquantel combinations ${ }^{(16)}$, daily micronutrients ${ }^{(5,31)}$ and delayed cord clamping ${ }^{(32)}$. Food fortification with micronised $\mathrm{Fe}$ improved children's $\mathrm{Fe}$ stores but did not increase Hb levels ${ }^{(2,33)}$.

\section{Discussion}

\section{Does the evidence support the anaemia policies?}

Several areas of agreement between policies were noted, especially in relation to Fe supplements and transfusion, and the need to treat co-morbidities. It was generally agreed that although there are continued concerns about potential increased risk of infections, as expressed at a WHO expert meeting in $2007^{(2)}$, Fe supplements are generally beneficial for treating anaemia especially in combination with other anaemia interventions. Fe given with foods was speculated to be safe, although administration as a fortificant has been questioned in terms of safety ${ }^{(34)}$. There was consensus that blood transfusion is indicated in children with severe anaemia who are clinically unstable. However, there were also examples of ambiguities within individual policies and inconsistencies between policies on key issues concerning anaemia diagnosis, treatment and prevention (Box 2). For example, the definitions for categories of anaemia severity were inconsistent, many policies did not specify the age groups for which the policy had been developed, and where target age groups were specified they varied between policies. As so few policies explicitly described the evidence on which they had been based, it was not possible to draw conclusions about whether publication of evidence was related to any revision of existing policies or to the development of new policies. Some of the contradictory findings in the evidence are likely to reflect differences in populations or population subgroups and their environment, which may limit the generalisability of some policies. However, for others, particularly those concerning diagnostics, there is insufficient available evidence to inform recommendations. The anomalies within and between policies, and in some cases their lack of generalisability, mean that information often cannot be pooled to harmonise the policies. Without more context-specific research to fill the current evidence gaps and to clarify any adaptations that are needed for specific contexts, clinicians will remain uncertain about selecting the best intervention for their own practice. The clear need to adapt global anaemia policies for each context highlights the importance of 
WHO headquarters restricting itself to making global anaemia policies and then WHO's regional offices can support local adaptations depending on the local context.

\section{Towards better policy making to reduce the burden of anaemia in African children and adolescents}

Evidence-based policy is difficult to achieve and health policies tend not to fully utilise available health research $^{(35)}$. Policy making is a multidirectional and convoluted process, and access to relevant research is only one component of a process involving interactions between researchers and policy makers in the context of the wider political, professional and social environments. Making good-quality policies for the diagnosis, treatment and prevention of anaemia is hampered by the complexity of anaemia. Anaemia is influenced by a variety of interdependent biological, environmental, social and political factors, so facilitating and harmonising contributions from a wide range of programmes and stakeholders will be challenging. Although WHO recommends that its guidelines should specify the duration of their validity, when they should be reviewed and which department will be responsible for initiating the review, most of the policies we identified did not have any indication of their duration of validity and few have undergone any documented process of updating. All the policies discussed in the present paper are currently available and therefore active policies. This plethora of potentially outdated policies with no process or timing for updating is one of the reasons for the conflicting information currently available about anaemia. By analysing the strengths and weaknesses of international policies concerning anaemia in children and adolescents in Africa, we have been able to identify and prioritise areas for further work. To address the weakest aspects of existing anaemia policies, attention should be focused on constituting appropriate guideline development and peer review groups and on more rigorous methods for updating and developing policies and incorporating evidence. A comprehensive review of existing research evidence concerning anaemia in African children is urgently needed so that knowledge gaps can be identified and prioritised and research commissioned to fill the gaps. Almost none of the current policies are specifically tailored to Africa, which raises concerns about the appropriateness of these policies to the African context. Research located in Africa and involvement of national stakeholders is essential to be able to answer context-specific questions concerning, for example, the risks and benefits of Fe supplementation where other infections are prevalent or in groups at high risk of anaemia such as those with HIV infection or sickle cell disease, and young infants. The safety of Fe interventions in malarious areas also has been reviewed by an expert panel of the US National Institutes of Health $(\mathrm{NIH})^{(36)}$. The Eunice Kennedy Shriver National Institute of Child Health and Human Development (NICHD) of the $\mathrm{NIH} / \mathrm{US}$ Department of Health and Human Services, in collaboration with partners, created the BOND Program to meet the growing need for the discovery, development and implementation of reliable and valid biomarkers to assess nutrient exposure, status, function and effect ${ }^{(37)}$. A primary aim is to harmonise processes for making decisions about what biomarkers are best for use in support of research, programme development and evaluation, and generation of evidence-based policy. The initiative responds to the need to examine the scientific basis for choosing appropriate methodologies to assess the role and impact of diet and nutrition on health and disease in populations and individuals, and to support development and evaluation of evidence-based programmes and policies to improve diet and nutrition. A central aspect of this is anaemia reduction.

Although the lack of policies concerning anaemia diagnosis is particularly worrying since effective anaemia screening and diagnosis is a critical entry point for many children into the health-care system, currently anaemia policy development is fragmented and those concerning Fe requirements are based largely on estimates of nutritional requirements from food intake studies in healthy Western children ${ }^{(38)}$. In areas with high infection exposure there is a need to identify specific Fe biomarkers which are not affected by concurrent inflammation. Hepcidin, an essential hormone which regulates $\mathrm{Fe}$ metabolism, may be useful for differentiating anaemia due to Fe deficiency from other causes ${ }^{(39)}$.

Policies are scattered among several programmes and organisations and access is through many different websites. This lack of harmonisation communicates mixed messages about anaemia diagnosis, treatment and prevention that make it difficult for health professionals to translate into rational actions on the ground. Although our review focuses on children, it is likely that a similar lack of harmonisation applies to policies concerning anaemia in other population subgroups such as pregnant women and the elderly. A critical step towards reducing the high burden of anaemia in African children should be the establishment of a single body within WHO responsible for devising and implementing a coherent approach to revising and developing anaemia policies and for making these policies easily accessible.

\section{Acknowledgements}

Sources of funding: This research received no specific grant from any funding agency in the public, commercial or not-for-profit sector. Conflicts of interest: All authors have completed the Unified Competing Interest form (http://www.icmje.org/coi_disclosure.pdf, available on request from the corresponding author) and declare: no support from any organisation for the submitted work; no 
financial relationships with any organisations that might have an interest in the submitted work in the previous 3 years; and no other relationships or activities that could appear to have influenced the submitted work. Ethical approval: This study did not require ethical approval/ patient consent as it is a review of policies and practices. Authors' contributions: M.H. undertook the literature and policy reviews, identified articles for inclusion in the study, contributed to design of the methods, and conducted the primary data analysis and interpretation. B.B. contributed to design of the methods, data analysis and interpretation, and provided guidance about inclusion/ exclusions of policies and evidence papers. I.B. devised the project, contributed to design of the methods, data analysis and interpretation, and provided guidance about inclusion/exclusions of policies and evidence papers. I.B. is guarantor. All authors reviewed drafts of the manuscript and approved the final version. Acknowledgements: The authors would like to thank Tanith Palmer, Research Assistant at Liverpool School of Tropical Medicine, for assistance with the preparation of this manuscript.

\section{Supplementary material}

To view supplementary material for this article, please visit http://dx.doi.org/10.1017/S1368980013003121

\section{References}

1. McLean E, Cogswell M, Egli I et al. (2009) Worldwide prevalence of anaemia, WHO Vitamin and Mineral Nutrition Information System, 1993-2005. Public Health Nutr 12, 444-454.

2. World Health Organization (2007) Conclusions and recommendations of the WHO Consultation on prevention and control of iron deficiency in infants and young children in malaria-endemic areas. Food Nutr Bull 28, 4 Suppl. 1, S621-S627.

3. Brouwers MC, Kho ME, Browman GP et al. (2010) AGREE II: advancing guideline development, reporting, and evaluation in health care. Prev Med 51, 421-424.

4. AGREE Next Steps Consortium (2009) The AGREE II Instrument. http://www.agreetrust.org (accessed May 2011).

5. Gera T, Sachdev HP \& Nestel P (2009) Effect of combining multiple micronutrients with iron supplementation on $\mathrm{Hb}$ response in children: systematic review of randomized controlled trials. Public Health Nutr 12, 756-773.

6. Gera T, Sachdev HP, Nestel P et al. (2007) Effect of iron supplementation on haemoglobin response in children: systematic review of randomised controlled trials. J Pediatr Gastroenterol Nutr 44, 468-486.

7. World Health Organization (2008) Essential Prevention and Care Interventions for Adults and Adolescents Living with HIV in Resource-Limited Settings. Geneva: WHO.

8. World Health Organization (2006) Guidelines for the Treatment of Malaria, 2nd ed. Geneva: WHO.

9. World Health Organization (2010) Antiretroviral Therapy for HIV Infection in Infants and Children: Towards Universal Access. Recommendations for a Public Health Approach: 2010 Revision. Geneva: WHO.

10. World Health Organization (2006) Antiretroviral Drugs for Treating Pregnant Women and Preventing HIV Infection in Infants: Towards Universal Access. Recommendations for a Public Health Approach. Geneva: WHO.

11. Chalco JP, Huicho L, Alamo C et al. (2005) Accuracy of clinical pallor in the diagnosis of anaemia in children: a meta-analysis. BMC Pediatr 5, 46.

12. Critchley J \& Bates I (2005) Haemoglobin colour scale for anaemia diagnosis where there is no laboratory: a systematic review. Int J Epidemiol 34, 1425-1434.

13. Ojukwu JU, Okebe JU, Yahav D et al. (2009) Oral iron supplementation for preventing or treating anaemia among children in malaria-endemic areas. Cochrane Database Syst Rev issue 3, CD006589.

14. Adetifa I \& Okomo U (2009) Iron supplementation for reducing morbidity and mortality in children with HIV. Cochrane Database Syst Rev issue 1, CD006736.

15. Meremikwu M \& Smith HJ (2000) Blood transfusion for treating malarial anaemia. Cochrane Database Syst Rev issue 2, CD001475.

16. Smith JL \& Brooker S (2010) Impact of hookworm infection and deworming on anaemia in non-pregnant populations: a systematic review. Trop Med Int Health 15, 776-795.

17. Cusick SE, Tielsch JM, Ramsan M et al. (2005) Short-term effects of vitamin $\mathrm{A}$ and antimalarial treatment on erythropoiesis in severely anemic Zanzibari preschool children. Am J Clin Nutr 82, 406-412.

18. Iannotti LL, Tielsch JM, Black MM et al. (2006) Iron supplementation in early childhood: health benefits and risks. Am J Clin Nutr 84, 1261-1276.

19. Baltussen R, Knai C \& Sharan M (2004) Iron fortification and iron supplementation are cost-effective interventions to reduce iron deficiency in four subregions of the world. J Nutr 134, 2678-2684.

20. Bojang KA, Milligan PJ, Conway DJ et al. (2010) Prevention of the recurrence of anaemia in Gambian children following discharge from hospital. PloS One 5, e11227.

21. Menendez C, Kahigwa E, Hirt R et al. (1997) Randomised placebo-controlled trial of iron supplementation and malaria chemoprophylaxis for prevention of severe anaemia and malaria in Tanzanian infants. Lancet 350, 844-850.

22. De-Regil LM, Suchdev PS, Vist GE et al. (2011) Home fortification of foods with multiple micronutrient powders for health and nutrition in children under two years of age. Cochrane Database Syst Rev issue 9, CD008959.

23. Zlotkin S, Newton S, Aimone AM et al. (2013) Effect of iron fortification on malaria incidence in infants and young children in Ghana: a randomized trial. JAMA 310, 938-947.

24. Soofi S, Cousens S, Iqbal SP et al. (2013) Effect of provision of daily zinc and iron with several micronutrients on growth and morbidity among young children in Pakistan: a cluster-randomised trial. Lancet 382, 29-40.

25. Berger J, Dyck JL, Galan P et al. (2000) Effect of daily iron supplementation on iron status, cell-mediated immunity, and incidence of infections in 6-36 month old Togolese children. Eur J Clin Nutr 54, 29-35.

26. Sazawal S, Black RE, Ramsan M et al. (2006) Effects of routine prophylactic supplementation with iron and folic acid on admission to hospital and mortality in preschool children in a high malaria transmission setting: communitybased, randomised, placebo-controlled trial. Lancet $\mathbf{3 6 7}$, 133-143.

27. Zimmermann MB, Chassard C, Rohner F et al. (2010) The effects of iron fortification on the gut microbiota in African children: a randomized controlled trial in Cote d'Ivoire. $\mathrm{Am}$ J Clin Nutr 92, 1406-1415.

28. Korenromp EL, Armstrong-Schellenberg JR, Williams BG et al. (2004) Impact of malaria control on childhood anaemia in Africa - a quantitative review. Trop Med Int Health 9, 1050-1065.

29. Kweku M, Liu D, Adjuik M et al. (2008) Seasonal intermittent preventive treatment for the prevention of 
anaemia and malaria in Ghanaian children: a randomized, placebo controlled trial. PloS One 3, e 4000.

30. Rohner F, Zimmermann MB, Amon RJ et al. (2010) In a randomized controlled trial of iron fortification, anthelmintic treatment, and intermittent preventive treatment of malaria for anemia control in Ivorian children, only anthelmintic treatment shows modest benefit. J Nutr 140, 635-641.

31. Smuts CM, Lombard CJ, Benade AJ et al. (2005) Efficacy of a foodlet-based multiple micronutrient supplement for preventing growth faltering, anemia, and micronutrient deficiency of infants: the four country IRIS trial pooled data analysis. J Nutr 135, issue 3, 631S-638S.

32. van Rheenen PF \& Brabin BJ (2006) A practical approach to timing cord clamping in resource poor settings. BMJ $\mathbf{3 3 3}$, 954-958.

33. Wegmuller R, Camara F, Zimmermann MB et al. (2006) Salt dual-fortified with iodine and micronized ground ferric pyrophosphate affects iron status but not hemoglobin in children in Cote d'Ivoire. J Nutr 136, 1814-1820.

34. Prentice AM, Verhoef H \& Cerami C (2013) Iron fortification and malaria risk in children. JAMA 310, 914-915.

35. Hanney SR, Gonzalez-Block MA, Buxton MJ et al. (2003) The utilisation of health research in policy-making: concepts, examples and methods of assessment. Health Res Policy Syst 1, 2.

36. Raiten DJ, Namaste S \& Brabin B (2011) Considerations for the safe and effective use of iron interventions in areas of malaria burden - executive summary. Int J Vitam Nutr Res 81, 57-71.

37. Raiten DJ, Namasté S, Brabin B et al. (2011) Executive summary - Biomarkers of Nutrition for Development: Building a Consensus. Am J Clin Nutr 94, issue 2, 633S-650S.

38. Lonnerdal B \& Kelleher SL (2007) Iron metabolism in infants and children. Food Nutr Bull 28, 4 Suppl., S491-S499.

39. Drakesmith H \& Prentice AM (2012) Hepcidin and the iron-infection axis. Science 338, 768-772.

40. Holzer BR, Egger M, Teuscher T et al. (1993) Childhood anemia in Africa: to transfuse or not transfuse? Acta Tropica 55, 47-51.

41. Alonzo González M, Menendez C, Font F et al. (2000) Costeffectiveness of iron supplementation and malaria chemoprophylaxis in the prevention of anaemia and malaria among Tanzanian infants. Bull World Health Organ 78, 97-107.

42. Okebe JU, Yahav D, Shbita $\mathrm{R}$ et al. (2011) Oral iron supplements for children in malaria-endemic areas. Cochrane Database Syst Rev issue 10, CD006589. 